\title{
Mechanism of Chip Formation Process at Grinding
}

\author{
Vyacheslav Shumyacher ${ }^{1 *}$, Sergey Kryukov ${ }^{1}$, Olga Kulik $^{1}$ and Xavier Kennedy ${ }^{2}$ \\ ${ }^{1}$ Volzhsky Polytechnic Institute (branch) of Volgograd State Technical University, 404121, Volzhsky, \\ Volgograd region, Engelsa street, 42a \\ ${ }^{2}$ Carborundum Universal Limited, No.655, T.H.Road, Thiruvottiyur Chennai-600019, India
}

\begin{abstract}
The mechanism of chip formation process at grinding is described, which involves a high-speed interaction of abrasive grain and metal, which leads to a concentration of thermal energy in front of the dispersing element (grain), causing a locally concentrated shift in the metal microvolume. In "abrasive grain - metal" contact a dissipative structure is formed which existence is supported by exchange of energy and substance with environment. Due to shock compression of the metal microvolume with abrasive grain, shock-wave heating is realized, initiating emission of electrons ionizing the lubricating cooling fluid in the zone of formation of side micro-scratches left by abrasive. The results obtained in the course of the research can be used to explain the mechanisms of chip formation, as well as the course of the physical and mechanical processes occurring on the surface layers of the grinded workpieces. By controlling chip formation processes at high-speed grinding, by optimally selecting the appropriate ratios between cutting speed and other processing parameters, a reduction in process thermal density can be achieved, which, with the highest productivity, will allow to obtain the required quality of the surface layer of the workpieces and a given dimensional accuracy.
\end{abstract}

\section{Introduction}

Abrasive processes in modern engineering occupy an important place. Formation of a given macro and microgeometry of working surfaces of critical parts is realized during abrasive machining, which often has no alternatives in development of technological processes. In this connection, information on chip formation mechanism at abrasive machining, which predetermines the quantitative and qualitative indicators of the process of forming a blank, is of scientific interest.

The works devoted to study of grinding processes made it possible to form requirements for quality and precision of processing [1,2], describe phenomena in the "tool - workpiece" contact $[3,4]$ at grinding, develop a model for interaction of abrasive grain and metal being processed $[5,6]$. At the same time, further study of the mechanochemical aspects of chip formation at abrasive machining is required to create a database for organizing an automated design system for abrasive processing technology in real production.

\footnotetext{
*Corresponding author: vms22@yandex.ru
} 
Grinding process is characterized by significant temperature gradients [7]. This stimulates the development of uncontrolled chemical reactions, diffusion processes, structural transformations in the treated surface, leading to thermal deformations, and subsequently microcracks.

At the heart of the overwhelming number of studies devoted to study of chip formation mechanism at grinding, the above ideas about the nature of processes occurring in the surface of the workpiece in contact with the grinding wheel prevail. The mechanism of separation of microchip with an abrasive grain is very rarely consecrated with a bias of microgeometric description of the profile of abrasive grain and roughness peak on the surface of the workpiece [8].

We have attempted from the standpoint of physicochemical mechanics to describe the mechanism of abrasive dispersion at grinding. Formation of microchip at grinding is adiabatic in nature and is governed by the ratio of the depth of introduction of abrasive grain $h$ to the radius of curvature of its vertex $\rho$ [9]. Due to the fact that the interaction time of abrasive grain and processed surface is $10^{-5}-10^{-4}$, there is reason to consider chip separation mechanism from the standpoint of the theory of high-velocity impact of solids. In the framework of proposed model of interaction of abrasive grain and material being processed at grinding [4], formation of microchip is the result of formation of significant energy before a moving abrasive. When processed metal passes through the front of the shock wave, it passes from solid to plastic state and then displaces abrasive grain to the side surfaces. Our study of the microstructures of metal on the matchmark surface left by abrasive grain showed presence of adiabatic shear bands, which according to [10] are the result of abrupt processes in the system of microshears and plastic rotations.

In accordance with [11-15], microshears and plastic rotations affect elastic and relaxation properties of the metal being processed.

Abrasive grain approximated by a ball is immersed in the metal to be processed to a depth $h$. The transfer rate of disturbances during the movement of abrasive grain is determined from the dependence [4]:

$$
J_{p}=\sqrt{J^{2}-2 J V \cos \alpha+V^{2}},
$$

where $J$ is fixed velocity of perturbation of the medium (metal),

$V$ is velocity of movement of abrasive grain,

$\alpha$ - is angle between the conduction velocity vector of disturbances of the surface abrasive grain and axis $O Z$ (Fig. 1).

The main property is conductivity of disturbance medium caused by the moving abrasive grain. The change in conductivity of the medium (metal) is the main cause of uneven removal of energy transferred by the grain in different parts of the medium.

Modeling the dependence (1) on a computer in a MATLAB simulation environment for different $V / J$ ratios is presented in Figure 2. 


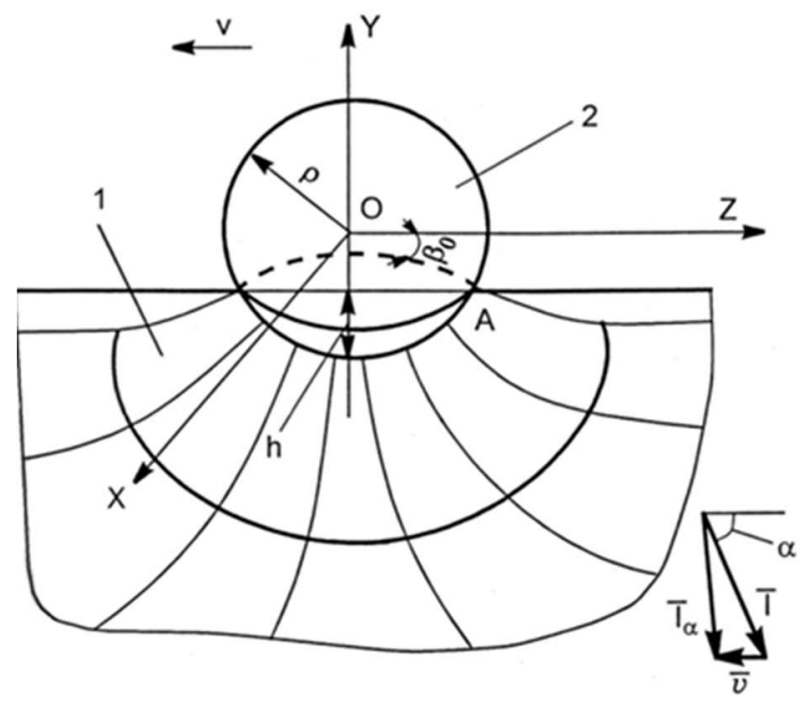

Fig.1. Model of interaction of abrasive grain and processed material at an arbitrary point in time
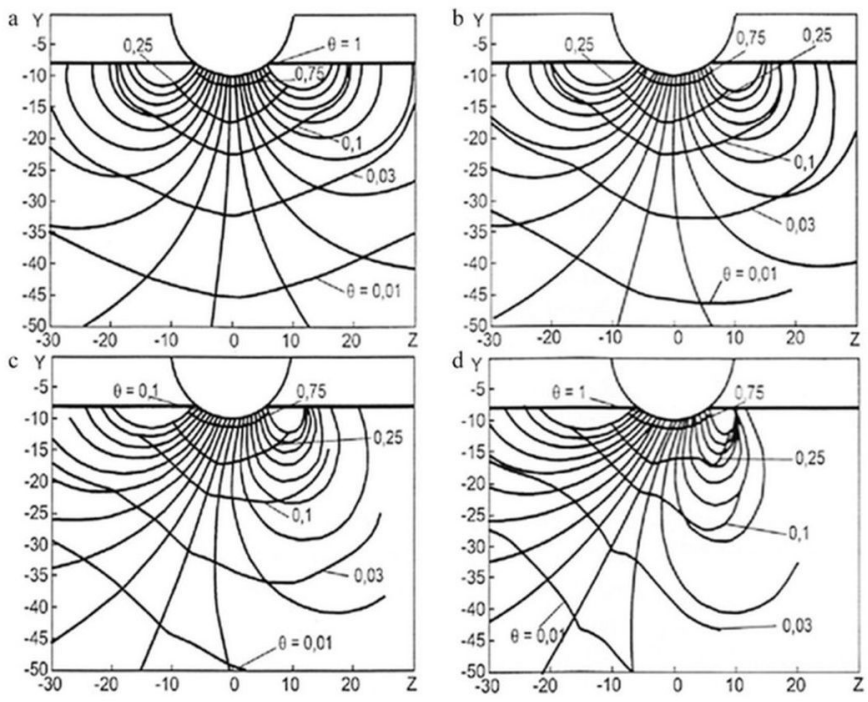

Fig.2. Stressed state in vicinity of the ball

From Figure 2 it follows that an increase in processing speed the geometry of rays changes. In the area of frontal part of the ball, the rays shift to its side. At a certain $V / J$ ratio (in this case, 0.45), a line appears waiting for a family of rays, which is the boundary when passing through which an abrupt change in parameters of the stressed state of the metal occurs. An increase in the depth of the introduction of abrasive grains into the metal, as well as an increase in its size, causes a shift in the wave formation condition (chip formation) towards lower speeds.

Increasing the speed of movement of abrasive grain leads to an increase in the length of the envelope (Fig. 2) and a clearer manifestation of shock wave. In the area of abrasive grain surface, the conditions for energy drain are improved, so the lines of the equally stressed state closely approach the abrasive. 
In this case, the pressure of abrasive grain on material is not enough to cause plastic deformation, so the pressure on abrasive increases. Growth of grain pressure on metal leads to its deformation. Accumulated energy in the lateral parts of abrasive grain is released as a burst in a favorable direction for its release, i.e. to the surface.

This phenomenon marks the beginning of a chip formation process. At the bottom of the matchmark left by abrasive grain, all the directions of metal flow are equivalent, therefore its structure changes as much as possible.

It is well known that cold-hardening at the bottom of the matchmark is significantly higher than in the area along its sides.

Shock-wave heating of metal being processed initiates emission of electrons, which interact with lubricating coolant significantly affect the dynamics of the processes in the "grain-metal" contact. An increase in ionization potential of the lubricating coolant reduces the coefficient of abrasive friction on the metal and sticking of micro chips.

\section{Conclusions}

Due to implementation of the above aspects of interaction of abrasive grain and the workpiece being processed, the chip formation process can be characterized as mechanochemical.

Chip formation mechanism can be represented by the following scheme:

- metal is forced out into the side zones of the micro scratch and its removal is carried out by the following abrasive grain;

- metal is displaced into piles on the sides of micro-scratches, the inertial forces tear it from the surface of the workpiece with formation of discharge chips;

- chip material at the time of separation breaks up into small particles of attractive forces which are not enough to overcome the forces of inertia.

Plastic particles pass into solid phase, coiling into hollow balls.

Based on the research we can draw the following conclusions:

- the basis of the mechanism of chip formation at grinding is shock-wave processes occurring when abrasive grain moves in the surface layer of the workpiece;

- accumulation of energy in front of abrasive grain leads to a decrease in pressure on the material necessary for its plastic deformation, which reduces the value of cutting force;

- increasing the depth of abrasive grain introduction shifts the conditions for formation of a shock wave in the direction of lower grinding speeds;

- individual micro chips are realized in three schemes, each of which is characterized by a specific form of chips.

\section{References}

1. V.F. Bezyazychniy, Surface Engineering: adj. to the journal: Reference book. Engineering magazine. 1, 9 (2001)

2. O.G. Bykadorova, V.M. Shumacher, Russian Engineering Research, 28, 836 (2008)

3. B.M. Brzhozovsky, A.V. Slavin, Bulletin of Saratov State Technical University, 4:60, $138(2011)$

4. A.V. Slavin, V.M. Shumyacher, Mechanical Engineering Technology, 1, 29 (2008)

5. V.M. Schumacher, A.V. Kadilnikov, Mechanical Engineering Technology, 4, 18 (2007)

6. V.M. Shumacher, A.V. Kadilnikov, Mechanical Engineering Technology, 5, 29 (2007)

7. S.A. Kryukov, N.V.Baidakova, LNME, 153, 1421 (2019)

8. D.G. Evseev, A.N. Salnikov, Grinding models. Theory and experiment (Moscow, Izvestia Publishing House, 2018)

9. O. G. Kulik, E. D. Illarionova, LNME, 153, 1457 (2019) 
10. L.W. Meyer, E. Staskewitsch, A. Burblies, Mechanics of Materials, 17, 175 (1994)

11. V. M. Shumyacher, A. V. Slavin, O. G. Kulik LNME, 153, 1443 (2019)

12. O.G. Kulik, V.A. Demenkov, Procedia Engineering, 206, 210 (2017)

13. O.G. Kulik, V.A. Demenkov, Procedia Engineering, 206, 216 (2017)

14. V.M. Shumyacher, A.V. Slavin, S.A. Kryukov, Procedia Engineering, 150, 916 (2016)

15. V.M. Shumyacher, A.V. Slavin, B.M. Brzhozowski, Procedia Engineering, 150, 920 (2016) 